\section{Successful long-term treatment with azacitidine in patient with chronic myelomonocytic leukemia}

\author{
Luka Čemažar, ${ }^{1}$ Helena Podgornik, $, 1,2$ \\ Njetočka Gredelj Šimec, ${ }^{1}$ Samo Zver ${ }^{1,3}$ \\ ${ }^{1}$ Department of Hematology, University \\ Clinical Centre Ljubljana; ${ }^{2}$ Faculty of \\ Pharmacy, University Clinical Centre \\ Ljubljana; ${ }^{3}$ Medical Faculty, University \\ Clinical Centre Ljubljana, Slovenia
}

\begin{abstract}
The purpose of this article was to present a case of successful long term treatment with azacitidine in patient with Chronic Myelomonocytic Leukemia (CMML) and discussing possible contributing factors for its long term efficacy. Data from our case were compared with similar data available in the literature. Effective treatment with azacitidine resulted in overall survival of 11 years 5 months and we showed that applying multiple cycles of treatment is feasible. Our patient received 71 cycles of treatment with total duration of 7 years and 3 months. Our report about a patient with CMML and a good clinical course revealed, that long term treatment with azacitidine is feasible in some patients. Initially low bone marrow blast count, a relatively small malignant CMML clone, reduction of spleen size and fast platelet response seemed to be factors determining long term response to treatment in our patient. More data on CMML treatment by Hypomethylating Agents and their analysis are needed in order to make firm conclusions.
\end{abstract}

\section{Introduction}

CMML is clinically diverse malignant clonal hematopoietic stem cell disorder characterized by persistent peripheral monocytosis, bone marrow dysplasia and with features of both myelodysplastic and myeloproliferative neoplasm. ${ }^{1}$ Generally, long term prognosis is poor with a median overall survival of 30 months and a relatively high risk of leukemic transformation, up to $30 \% .^{2}$ Hypomethylating agents belong to a class of drugs which inhibit DNA methyltransferase and consequently reduce DNA methylation. Utilization of HMA in CMML has been reported with seemingly unsatisfying results with overall response rates between 30 and $60 \%$ and median overall survival between 12 and 37 months. ${ }^{3,4}$ Previously only a few predictive factors (increased bone marrow blast count and proliferative features) were found to be associated with shorter survival. ${ }^{5}$ Surprisingly only age, but none of the other disease characteristics, was a prediction for a response. ${ }^{6}$

In this case report, we describe an effective long term use of azacitidine in a patient with CMML and tried to find factors, which may contribute to long term azacitidine efficacy.

\section{Case Report}

A 58-years-old male patient presented with a moderate anemia (hemoglobin 93 $\mathrm{g} / \mathrm{L}$ ) and thrombocytopenia (platelet count $\left.112 \times 10^{9} / \mathrm{L}\right)$. Because he was clinically asymptomatic, we decided for a "watch and wait" approach with a regular outpatient visits. His blood count remained stable without treatment for another 3 years. Later on, a gradual increase in leukocyte count was noticed with predominant monocytosis. Bone marrow work up morphologically revealed CMML with $6 \%$ of blast cells. Cytogenetics showed a normal karyotype. For the four following years, he did not need any therapy. In the meantime, he suffered from progressive joint and muscles pain and had platelet count ranging between $50 \times 10^{9} / \mathrm{L}$ and $140 \times 10^{9} / \mathrm{L}$. Fluctuations in platelet count may have suggested accompanied autoimmune mechanism, immune thrombocytopenia. $^{7}$

At that point the treatment was considered due to increased leucocyte count. His blood count was: WBC $68.5 \times 10^{9} / \mathrm{L}$, Hemoglobin $101 \mathrm{~g} / \mathrm{L}$, and platelets $101 \times 10^{9} /$ L. Blood differential showed: $40.8 \times 10^{9} / \mathrm{L} \quad$ neutrophils $\quad(59.6 \%)$, $23.1 \times 10^{9} / \mathrm{L}$ monocytes $(33.8 \%), 4.2 \times 10^{9} / \mathrm{L}$ lymphocytes $(6.1 \%)$ without immature granulocytes or blasts. The patient did not have any clinical symptoms, spleen size was $2 \mathrm{~cm}$ below the left costal margin. After 4,5 years from the initial diagnosis, reexamined bone marrow cytogenetics revealed balanced translocation between homologous chromosomes $11, \mathrm{t}(11 ; 11)(\mathrm{p} 15 ; \mathrm{q} 11)$ in otherwise small clone of metaphases, $15 \%$. The aberration is not recurrent and was not described in CMML before. Based on MD Anderson Prognostic Scoring System (MDAPS) for CMML, the patient was in an intermediate- 2 risk group. ${ }^{8}$ Patient began treatment with azacitidine $75 \mathrm{mg} / \mathrm{m}^{2}$ daily 7 days per month. After 2 cycles, treating hematologist tampered the dose to a fixed $75 \mathrm{mg}$ for following 4 cycles, due to neu-
Correspondence: Samo Zver, University Medical Centre Ljubljana, Department of Hematology, Zaloška cesta 7, 1000 Ljubljana Slovenia

Tel.: +386 15223135 - Fax: +3861522 2727 . E-mail: samo.zver@kclj.si

Key words: chronic myelomonocytic leukemia; azacitidine; long-term treatment.

Contributions: the authors contributed equally.

Conflict of interest: the authors declare no conflict of interest

Funding: none.

Ethics approval and consent to participate: Both consent and approval have been obtained.

Availability of data and materials: Data and materials available within the text.

Received for publication: 12 March 2020

Accepted for publication: 30 Ocober 2020.

This work is licensed under a Creative Commons Attribution-NonCommercial 4.0 International License (CC BY-NC 4.0).

(C) Copyright: the Author(s), 2020

Licensee PAGEPress, Italy

Hematology Reports 2020; 12:8537

doi:10.4081/hr.2020.8537

tropenia. From cycle 7 onwards he again received a full dose.

Although he never achieved complete remission, we observed clear hematological response and clinical improvement. We evaluated the response according to "International consortium proposal of uniform response criteria for (MDS/MPN)". ${ }^{3}$ Clinical improvement was observed after 4 cycles of azacitidine. Besides monocyte count decreased to $1.4 \times 10^{9} / \mathrm{L}$, platelets increased to $250 \times 10^{9} / \mathrm{L}$ and he became red cells transfusion independent, with hemoglobin level $110 \mathrm{~g} / \mathrm{L}$. Dynamic of monocyte and platelet counts were dependent upon azacitidine administrations (Figure 1). Eleven azacitidine cycles resulted in a partial remission.

After 37 cycles (duration of azacitidine treatment 3 years and 9 months), bone marrow histology was typical for CMML, without blasts. Cytogenetics revealed two new clones of comparable size ( $25 \%$ of cells) while the initial clone observed at the diagnosis has disappeared. Both clones had a partial trisomy for long arm of chromosome 1. Besides the loss of chromosome Y, there was an additional derivative chromosome 1 lacking the whole short arm. In the second 
clone additional copy of long arm of chromosome 1 was translocated to short arm of chromosome 21. Considering all aberrations, he acquired a complex karyotypeazacitidine treatment continued for 71 cycles (total duration of azacitidine treatment 7 years and 3 months), until progression to secondary acute myeloid leukaemia occurred. Due to the deterioration of the disease, diagnostic work up was not repeated. He was treated symptomatically and died shortly after transformation was confirmed. After being diagnosed, he lived 11 years and 5 months.

\section{Discussion}

Latest World Health Organization (WHO) classification of myeloid neoplasms defines CMML as a clonal hematopoietic stem cell disorder characterized by the presence of sustained (i.e., $>3$ months) Peripheral Blood (PB) monocytosis $\left(\geq 1 \times 10^{9} / \mathrm{L}\right.$ and monocytes being $\geq 10 \%$ of total white blood cell count) along with dysplastic features in the bone marrow. ${ }^{1} \mathrm{CMML}$ is heterogenic in nature and patients' clinical course and treatment outcomes are variable. Several prognostic models, have been developed. ${ }^{2,3}$ For risk stratification, we used MD Anderson Prognostic Scoring System (MDAPS). Otherwise different, similar prognostic models offer comparable results. ${ }^{9}$ Nevertheless, our patient fitted in the intermediate-2-risk group. The latter means a median overall survival of 9 months, although it should be stressed out, that the scoring system was designed before the era of hypomethylating agents (HMA). ${ }^{10}$

Cytogenetic abnormalities are present in one-third of the patients with CMML. Firstly, we detected loss of the chromosome $\mathrm{Y}$, which is well known recurrent aberration in MDS. ${ }^{9}$ On the other hand, other aberrations observed during the course of the disease, i.e. partial trisomy of chromosome 1 is less frequently described in MDS. New cytogenetics abnormalities are a sign of a clonal evolution and this has negative prognostic impact on the overall survival. ${ }^{11}$ Although the later onset of clonal evolution has a better outcome than an early one. ${ }^{11}$ According to Mayo Clinic -French Consortium Study there are three cytogenetic risk categories, high (complex and monosomal karyotypes), intermediate and low [normal, sole -Y and sole $\operatorname{der}(3 q)$ ], with adjusted median survival of 3,20 and 41 months. ${ }^{12}$ Although our patient started HMA treatment in cytogenetic intermediate risk group, latter disappearance of the initial clone with non-recurrent $\mathrm{t}(11 ; 11)$ probably points to relatively benign cytogenetic aberration. However, by clonal evolution the patient acquired a complex high risk karyotype and dyed shortly after that.

Several studies and clinical trials tested efficacy of both azacitidine and decitabine and they revealed overall response rates in the range of $30-60 \%$ and a median Overall
Survival (OS) between 12 to 37 months. $3,4,11,12$ Based on latest findings, azacitidine was licensed for non-proliferative CMML-2, whereas there are limited data for proliferative CMML subtypes. ${ }^{4}$ According to one of the recent studies the overall response rate for azacitidine treatment was $20 \%$ and the majority of patients achieved response after a median of 3 cycles of treatment. Duration of the response varied between 3 and 24 months. ${ }^{13}$ In our case, although the patient had proliferative features of CMML, OS was 11 years and 5 months, which is far longer than OS mentioned in previous publications. Patient's quality of life on azacitidine was seemingly unimpaired, putting aside some minor respiratory infections and low grades azacitidine related adverse reactions.

When trying to understand long period of azacitidine efficacy in our case, other authors stated, that bone marrow blasts higher than $10 \%$ and proliferative features of the disease as an increased WBC and splenomegaly were associated with shorter survival and surprisingly, responders were older $(71.4 \mathrm{y})$ than non-responders $(66.5 \mathrm{y}) .^{5}$ At treatment initiation our patient was 66 years old, had low bone marrow blasts $(6 \%)$, an increased WBC and an enlarged spleen. Low level of bone marrow infiltration with blasts, might be in relation with an initially relatively small malignant CMML clone. Anyway, karyotyping with its low number of metaphases is not an optimal method. Reduction of spleen size during the treatment

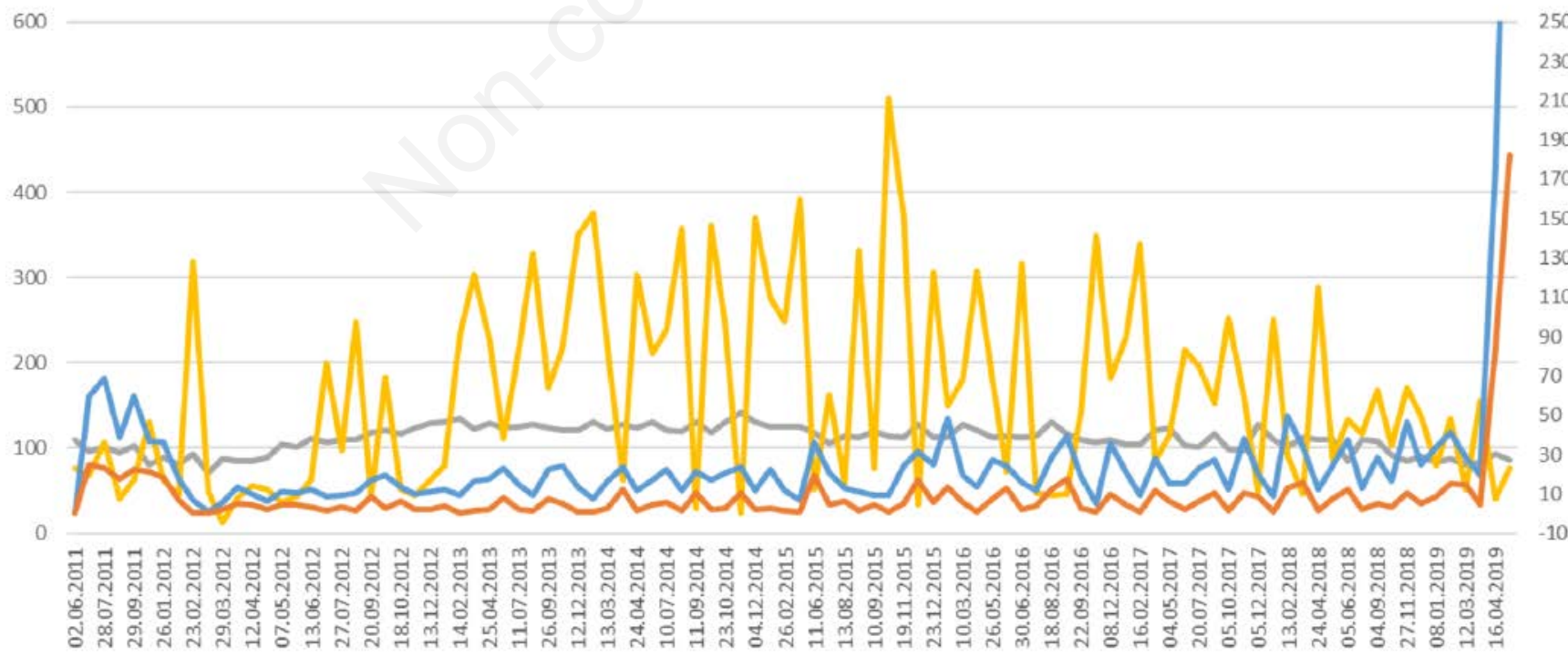

Hemoglobin Platelets Leukocytes Monocytes

Figure 1. Correlation between platelets count and the monocyte count in response to azacitidine treatment. On the left side of the $y$ axis of the Chart 1 there are numbers which represent values for the hemoglobin level and the platelets count and on the right side of $y$-axis there are values for the monocytes and leukocytes count. There are signs of correlation between an increase of platelets count and the decrease of monocyte count in response to azacitidine treatment. 
could be another cornerstone on path to continuous improvement. Of interest, one report stated, that doubling of platelets after first round of azacitidine was a positive predictor of overall survival. The latter was observed also in our patient. ${ }^{14}$

In order to be more precise, we should have determined patient's molecular genetic profile to find any mutations associated with a better outcome. So far only ASXL1 and SETBP1 mutations were found to have negative prognostic impact on overall survival in CMML. The use of Next-Generation Sequencing (NGS) would probably give us in future more definite answers on prognosis for our patients. ${ }^{15,16}$ Unfortunately, we had limited access to NGS at that time.

Observed serious adverse effects were consistent with already published ones. ${ }^{17}$ Myelosuppression and infections were mostly observed and are responsible for treatment delay. ${ }^{13}$ We reduced azacitidine dose due to neutropenia several times. And postponed some of the applications because of minor respiratory infections which normally occurred in $2.5 \%$ of azacitidine treated patients. ${ }^{18}$ Among more serious side effects, our patient experienced progression of ischemic heart disease which may be attributed to azacitidine. That was not possible to rule out and it manifested clinically after 60 cycles of treatment. Although we can not prove a direct relation between azacitidine and cardiac toxicity, it is highly likely as there were case reports describing possible connection between HMA and acute myocarditis, pericarditis and cardiomyopathy. ${ }^{19-21}$ Although still rare more common cardiovascular side effects mentioned are chest discomfort, peripheral edema and heart murmurs. ${ }^{20}$

In conclusion, progression of CMML to acute leukemia occurred as a result of disease natural clinical course. CMML patients have $18 \%$ to $63 \%$ chance of progress into AML during 5 years. ${ }^{22}$ Acute leukemia free interval was in our patient almost identical with his OS; 11 years and three months. The latter is much longer than a median acute leukemia free survival of 27.4 months reported for CMML patients within intermediate risk group. ${ }^{23}$ Nevertheless transformation to acute leukemia has a dismal prognosis with poor outcomes. ${ }^{24}$

\section{Conclusions}

We may conclude, that this was the case report of a CMML patient, with extreme good clinical course. And that is why it should be analyzed in detail in order to understand the malignant disease better.

\section{References}

1. Arber DA, Orazi A, Hasserjian R, et al. The 2016 revision to the World Health Organization classification of myeloid neoplasms and acute leukemia. Blood 2016 19;127:2391-405.

2. Clara JA, Sallman DA, Padron E. Clinical management of myelodysplastic syndrome/myeloproliferative neoplasm overlap syndromes. Cancer Biol Med 2016;13:360-72.

3. Patnaik MM, Tefferi A. Chronic myelomonocytic leukemia: 2018 update on diagnosis, risk stratification and management. Am J Hematol 2018;93:824-40.

4. Solary E, Itzykson R. How I treat chronic myelomonocytic leukemia. Blood 2017 13;130:126-36.

5. Adès L, Sekeres MA, Wolfromm A, et al. Predictive factors of response and survival among chronic myelomonocytic leukemia patients treated with azacitidine. Leuk Res 2013;37:609-13.

6. Almeida A. Modifying disease in CMML: Who responds to Azacitidine? Leuk Res. 2013;37:603-4.

7. Montoro J, Gallur L, Merchán B, et al. Autoimmune disorders are common in myelodysplastic syndrome patients and confer an adverse impact on outcomes. Ann Hematol 2018;97:1349-56.

8. Beran M, Wen S, Shen Y, et al. Prognostic factors and risk assessment in chronic myelomonocytic leukemia: validation study of the M.D. Anderson Prognostic Scoring System. Leuk Lymphoma. 2007;48:1150-60.

9. Padron E, Garcia-Manero G, Patnaik MM, et al. An international data set for CMML validates prognostic scoring systems and demonstrates a need for novel prognostication strategies. Blood Cancer J 2015;5:e333.

10. Onida F, Kantarjian HM, Smith TL, et al. Prognostic factors and scoring systems in chronic myelomonocytic leukemia: a retrospective analysis of 213 patients. Blood 2002;99:840-9.

11. Thorpe M, Montalvão A, Pierdomenico F, Moita F, Almeida A. Treatment of chronic myelomonocytic leukemia with 5-Azacitidine: a case series and literature review. Leuk Res 2012;36:1071-3.

12. Drummond MW, Pocock C, Boissinot $\mathrm{M}$, et al. A multi-centre phase 2 study of azacitidine in chronic myelomonocytic leukaemia. Leukemia 2014;28:1570-2.

13. Helbig G, Chromik K, Woźniczka K, et al. Real life data on efficacy and safety of azacitidine therapy for myelodysplastic syndrome, chronic myelomono- cytic leukemia and acute myeloid leukemia. Pathol Oncol Res 2019;25: 1175-80.

14. Van der Helm LH, Alhan C, Wijermans PW, et al. Platelet doubling after the first azacitidine cycle is a promising predictor for response in myelodysplastic syndromes (MDS), chronic myelomonocytic leukaemia (CMML) and acute myeloid leukaemia (AML) patients in the Dutch azacitidine compassionate named patient programme. Br J Haematol 2011;155:599-606.

15. Sallman D, Padron E, Vaupel C, et al. Prognostic Impact of ASXL1 Mutations in MDS and CMML. Blood 2015; 126:1673.

16. Laborde RR, Patnaik MM, Lasho TL, et al. SETBP1 mutations in 415 patients with primary myelofibrosis or chronic myelomonocytic leukemia: independent prognostic impact in CMML. Leukemia 2013;27:2100-2.

17. San Miguel Amigo L, Franco Osorio R, Mercadal Vilchez S, Martínez-Francés A. Azacitidine adverse effects in patients with myelodysplastic syndromes. Adv Ther 2011;28:6-11.

18. Vidaza $25 \mathrm{mg} / \mathrm{mL}$ powder for suspension for injection. Product information. Accessed: 2019 Jul 27. Available from: https://www.ema.europa.eu/en/documents/product-information/vidazaepar-product-information_en.pdf.

19. Bibault J-E, Cambier N, Lemahieu J-M, et al. Acute myocarditis induced by hypomethylating agents. J Clin Oncol Off J Am Soc Clin Oncol 2011;29:e4112.

20. De C, Phookan J, Parikh V, et al. Decitabine induced transient cardiomyopathy: a case report. Clin Med Insights Oncol 2012;6:325-9.

21. Newman M, Malla M, Gojo I. Azacitidine-induced pericarditis: a case series. Pharmacother 2016;36:443-8.

22. Liu H, Cheng J, Zhao L, et al. Outcome of patient with high-risk chronic myelomonocytic leukemia, treated with decitabine prior to transformation to acute myeloid leukemia: A case report. Oncol Lett 2018;15:7132-8.

23. Itzykson R, Kosmider O, Renneville A, et al. Prognostic score including gene mutations in chronic myelomonocytic leukemia. J Clin Oncol 2013;31:242836.

24. Niscola P, Tendas A, Scaramucci L, et al. Acute myeloid leukemia arising from chronic myelomonocytic leukemia during hypomethylating therapy. Blood Res 2014;49:65-6. 